\title{
MPMI ACKNOWLEDGMENT OF REVIEWERS
}

The success of Molecular Plant-Microbe Interactions depends on the quality of manuscripts submitted by authors and on the care and competence with which they are reviewed. It is the policy of the Editorial Board to solicit reviews of manuscripts from specialists most qualified to review them. In addition to members of the Editorial Board, the individuals listed below provided constructive critical reviews of one or more manuscripts during the past year. Their names are published here in grateful appreciation for their contributions to the journal.

C. Alabouvette, UMR INRA Université de Bourgogne, Dijon, France

J. R. Alfano, University of Nebraska, Lincoln, NE, U.S.A.

C. Allen, University of Wisconsin, Madison, WI, U.S.A.

A. Alvarez-Morales, Cinvestav-IPN, Irapuato, GTO, Mexico

J.-M. Ane, University of California, Davis, CA, U.S.A.

A. Bailey, Bristol University, Clifton, Bristol, U.K.

I. Barash, Tel-Aviv University, Tel Aviv, Israel

C. Baron, McMaster University, Hamilton, ON, Canada

P. Bauer, Leibniz Institute, Gatersleben, Germany

T. J. Baum, Iowa State University, Ames, IA, U.S.A.

D. Bell-Pedersen, Texas A\&M University, College Station,

TX, U.S.A.

C. Bender, Oklahoma State University, Stillwater, OK, U.S.A.

A. Bent, University of Wisconsin, Madison, WI, U.S.A.

J. A. E. Benen, Wageningen University, Wageningen, The Netherlands

A. Bent, University of Wisconsin, Madison, WI, U.S.A.

C. Bergmann, University of Georgia, Athens, GA, U.S.A.

J. Beynon, Horticulture Research International, Wellesbourne, Warwickshire, U.K.

D. Bird, North Carolina State University, Raleigh, NC, U.S.A.

T. Bisseling, Wageningen University, Wageningen, The Netherlands

V. Blok, Scottish Crop Research Institute, Invergowrie, Dundee, U.K.

A. J. Bogdanove, Iowa State University, Ames, IA, U.S.A.

H. Bohnert, University of Illinois, Urbana, IL, U.S.A.

J. F. Bol, Leiden University, Leiden, The Netherlands

T. Boller, University Basel, Basel, Switzerland

D. Borthakur, University of Hawaii, Honolulu, Hawaii

H. Bothe, Universität zu Köln, Koln, Germany

C. Boucher, CNRS-INRA, Castanet Tolosan, France

G. Bryan, Scottish Crop Research Institute, Invergowie, Dundee, U.K.

I. Carbone, North Carolina State University, Raleigh, NC, U.S.A.

F. Cervone, Universita' di Roma "La Sapienza", Roma, Italy

T. Charles, University of Waterloo, Waterloo, ON, Canada

A. Chatterjee, Universiry of Missouri, Columbia, MO, U.S.A. Italy

M. Chiurazzi, Intl Inst of Genetics \& Biophysics, Naples,

S. Clemens, Leibniz Institute, Weinberg, Halle, Germany

D. B. Collinge, Royal Veterinary and Agricultural University, Frederiksberg C, Denmark

A. Collmer, Cornell University, Ithaca, NY, U.S.A.

R. M. Cooper, University of Bath, Bath, U.K.

D. L. Coplin, Ohio State University, Columbus, OH, U.S.A.

D. Cosgove, Penn State University, University Park, PA, U.S.A.

V. Crane, Pioneer Hi-Bred International, Johnston, IA, U.S.A.
M. Crespi, CNRS, Gif sur Yvette, France

J. Cullimore, INRA-CNRS, Castanet-Tolosan, France

D. Cuppels, Agriculture and Agri-food Canada, London, ON, Canada

K. Czymmek, Delaware Biotechnology Institute, Newark, DE, U.S.A.

W. D'Haeze, University of Georgia, Athens, GA, U.S.A.

C. D'Souza, The University of British Columbia, Vancouver,

B.C., Canada

D. Dalton, Reed College, Portland,OR, U.S.A.

E. Davis, North Carolina State University, Raleigh, NC, U.S.A.

M. Davis, Tropical Research and Education Center, Homestead, FL, U.S.A.

D. A. Day, University of Western Australia, Crawley, WA, Australia

F. Dazzo, Michigan State University, East Lansing, MI, U.S.A.

J. De Boer, Laboratory of Plant Breeding, Wageningen University, Wageningen, The Netherlands

R. De Mot, K.U. Leuven, Heverlee, Belgium

M. Delledonne, Universita' degli Studi di Verona, Verona, Italy

M. Deom, University of Georgia, Athens, GA, U.S.A.

G. Desbrosses, Université Montpellier II, Montpellier, France

M. B. Dickman, University of Nebraska, Lincoln, NE, U.S.A.

S. W. Ding, University of California, Riverside, CA, U.S.A.

B. Ding, Ohio State University, Columbus, OH, U.S.A.

M. Dixon, University of Southampton, Southampton, U.K.

V. V. Dolja, Oregon State University, Corvallis, OR, U.S.A.

M. Dow, National University of Ireland, Cork Ireland

A. Downie, John Innes Institute, Colney Lane, Norwich, U.K.

M. Dunn, Universidad Nacional Autonoma de Mexico, Cuernavaca, Morelos, Mexico

D. Ebbole, Texas A\&M University, College Station, TX, U.S.A.

B. Emerson, University of East Anglia, Norwich, U.K.

G. Endre, Institute of Genetics, Szeged, Hungary

M. Farman, University of Kentucky, Lexington, KY, U.S.A.

S. Farrand, University of Illinois, Urbana, IL, U.S.A.

B. Feys, Imperial College London, London, U.K.

T. M. Finan, McMaster University, Hamilton, ON, Canada

C. Foyer, Rothamsted Research, Harpenden, Herts, U.K.

P. Franken, Institute for Vegetable and Ornamental Crops, Grossbeeren, Germany

R. Fray, University of Nottingham, Loughborough, U.K.

R. French, University of Nebraska, Lincoln, NE, U.S.A.

M. Fromm, University of Nebraska, Lincoln, NE, U.S.A.

P. Gamas, INRA-CNRS, Castanet-Tolosan, France

F. Garcia-Arenal, Ciudad Universitaria, Madrid, Spain

W. Gassman, University of Missouri, Columbia, MO, U.S.A. 
S. Gelvin, Purdue University, West Lafayette, IN, U.S.A.

R. Genger, University of Wisconsin, Madison, WI, U.S.A

S. Genin, INRA, INRA-CNRS,Castanet-Tolosan, France

T. L. German, University of Wisconsin, Madison, WI, U.S.A.

R. Gilbertson, University of California, Davis, CA, U.S.A.

D. G. Gilchrist, University of California, Davis, CA, U.S.A.

K. Giller, Wageningen University, Wageningen, The Netherlands

J. Glazebrook, University of Minnesota, St. Paul, MN, U.S.A.

A. E. Glenn, USDA ARS, Athens, GA, U.S.A.

S. B. Goodwin, Purdue University, West Lafayette, IN, U.S.A.

M. Gottfert, Institut für Genetik, Dresden, Germany

J. Green, University of Birmingham, Birmingham, U.K.

P. Gresshoff, University of Queensland, Brisbane, St. Lucia,

Australia

B. Gronenborn, ISV-CNRS, Gif sur Yvette, France

R. Geurts, Wageningen University, Wageningen, The Netherlands

S. Gurr, University of Oxford, Oxford, U.K.

D. S. Guttman, University of Toronto, Toronto, ON, Canada

L. J. Harris, Agriculture and Agri-Food Canada, Ottawa, Canada

M. Harrison, Boyce Thompson Institute for Plant Research, Ithaca, NY, U.S.A.

A. Hartman, GSF-Research Center, Neuherberg/Munich, Germany

M. Hawes, University of Arizona, Tucson, AZ, U.S.A.

M. Hayashi, Osaka University, Osaka, Japan

S. Y. He, Michigan State University, East Lansing, MI, U.S.A.

J. Helder, Wageningen University, Wageningen, The Netherlands

H. Hirt, Kansas State University, Manhattan, KS, U.S.A.

M. Höfte, Ghent University, Gent, Belgium

S. Hogenhout, Ohio State University, Wooster, OH, U.S.A.

M. Holsters, UG/VIB2, Zwijnaarde (Gent), Belgium

Y.-M. Hou, Syngenta Biotechnology, Inc., Research Triangle Park, NC, U.S.A.

N. Hugouvieux-Cotte-Pattat, UMR 5122 CNRS-UCB-

INSA, Villeurbanne, France

R. Hull, John Innes Centre, Colney, Norwuch, United Kindgom

S. Hutcheson, University of Maryland, College Park, MD, U.S.A.

C. F. Hwang, University of California, Davis, CA, U. S.A.

I. Hwang, Seoul National University, Seoul, South Korea

M. Hynes, University of Calgary, Calgary, Alberta, Canada

R. Innes, Indiana University, Bloomington, IN, U.S.A.

M. Ishikawa, National Institute of Agrobiological Sciences,

Tsukuba, Japan

A. Jackson, University of California, Berkeley, CA, U.S.A.

E. James, University of Dundee, Dundee, U.K.

J.-S. Jeon, Kyung Hee University, Kyungi, Korea

Y. Jia, USDA ARS DB NRRC, Stuttgart, AR, U.S.A.

A. W. B. Johnston, University of East Anglia, Norwich, U.K.

A. Jones, Imperial College London, Wye, Ashford, U.K.

J. B. Jones, University of Florida, Gainesville, FL, U.S.A.

D. A. Jones, Australian National University, Canberra ACT, Australia

J. Jones, Scottish Crop Research Institute, Invergowrie, Dundee, U.K.

M. H. A. J. Joosten, Wageningen University, Wageningen, The Netherlands

H. Judelson, University of California, Riverside, CA, U.S.A.

P. Kachroo, University of Kentucky, Lexington, KY, U.S.A.

I. Kaloshian, University of California, Riverside, CA, U.S.A.
S. Kang, The Pennsylvania State University, University Park, PA, U.S.A.

E. Kannenberg, The University of Georgia, Athens, GA, U.S.A.

F. Katagiri, University of Minnesota, St. Paul, MN, U.S.A.

P. Katinakis, Agricultural University of Athens, Athens, Greece

C. Keel, Universite de Lausanne, Lausanne-Dorigny, Switzerland

C. Kenerley, Texas A\&M University, College Station, TX, U.S.A.

C. Kennedy, University of Arizona, Tucson, AZ, U.S.A

C. Kistner, Deutsche Forschungsgemeinschaft DFG, Bonn, Germany

T. Klein, DuPont Crop Genetics, Wilmington, DE, U.S.A.

W. Knogge, The University Of Adelaide, Adelaide SA, Australia

F. Krajinski, University Hannover, Hannover, Germany

J. Kronstad, The University of British Columbia, Vancouver, B.C., Canada

L. Krusell, Max Planck Institute of Molecular Plant Physiology, Golm, Germany

C. P. Kubicek, Institute of Chemical Engineering, Vienna, Austria

H. Kuester, Universität Bielefeld, Bielefeld, Germany

B. Kunkel, Washington University, St. Louis, MO, U.S.A.

T. Lahaye, Institut fuer Genetik, Halle (Salle), Germany

M. Latijnhouwers, Scottisch Crop Research Institute, Dundee, U.K.

C. Lawrence, Virginia Bioinformatics Institute, Virginia Tech, Blacksburg, VA, U.S.A.

D. Le Rudulier, University of Nice, Parc Valrose, Nice, France

J. R. Leadbetter, California Institute of Technology, M/C, Pasedena, CA, U.S.A.

S. Leisner, The University of Toledo, Toledo, OH, U.S.A

D. Leister, Max-Planck Institute for Plant Breeding Research, Cologne, Germany

S. E. Lindow, University of California, Berkeley, CA, U.S.A.

K. Lindsey, University of Durham, Durham, U.K.

J. Loper, USDA ARS, Corvallis, OR, U.S.A.

M. Lorito, Universita Degli Studi Di Napoli "Federico II", Portici, Italy

J. Ludwig-Mueller, Technische Universitaet Dresden, Dresden, Germany

J. W. Mansfield, Wye College, Wye, Ashford, U.K.

F. Martin, Inst Natl Recherche Agronomique, Champenoux, France

U. Mathesius, The Australian National University, Canberra, Australia

A. Maule, John Innes Institute, Colney Lane, Norwich, U.K.

R. McIntosh, Institute Cobbitty, Camden, Australia

J.-P. Metraux, University of Fribourg, Fribourg, Switzerland

A. J. Michael, Institute of Food Research, Norwich

Research Park, Colney, U.K.

T. K. Mitchell, North Carolina State University, Raleigh, NC, U.S.A.

P. Moffett, Boyce Thompson Institute for Plant Research, Ithaca, NY, U.S.A.

I. Moore, University of Oxford, Oxford, U.K.

S. Morozov, Moscow State University, Russia

J. Morrissey, University College Cork, Cork, Ireland

P. Munsch, University of Oulu, University of Oulu, Finland

L A. J. Mur, University of Wales, Aberystwyth, Wales, U.K.

S. Muthukrishnan, Kansas State University, Manhattan, KS, U.S.A.

R. S. Nelson, Samuel Roberts Noble Foundation, Inc., Ardmore, OK, U.S.A. 
A. Niebel, INRA CNRS LBMRPM, Castanet Tolosan, France

D. Noel, Marquette University, Milwaukee, WI, U.S.A.

T. Nuernberger, Eberhard-Karls Univ Tubingen, Tübingen, Germany

O. Nybroe, Royal Veterinary and Agricultural University, Frederiksberg C, Denmark

S.i Oide, Cornell University, Ithaca, NY, U.S.A.

R. J. O'Connell, CNRS-UPS, Auzeville, France

G. Oldroyd, John Innes Centre, Norwich, U.K.

J. Oliveira, Universidade Estadual Paulista - Jaboticabal, São Paulo, Brazil

R. Oliver, Murdoch University, Perth 6150, Western Australia

R. Omarov, Texas A\&M University, College Station, TX, U.S.A.

K. Oparka, Scottish Crop Research Institute, Scotland, U.K.

N. Orange, Universite de Rouen, Evreux, France

M. Orbach, University of Arizona, Tucson, AZ, U.S.A.

A. Osbourn, John Innes Centre, Norwich, U.K.

T. Ouellet, ECORC-AAFC, Ottawa, ON, Canada

M. Gjedde Palmgren, The Royal Veterinary and Agricultural

University, Frederiksberg C, Copenhagen, Denmark

T. Palva, University of Helsinki, Helsinki, Finland

U. Paszkowski, Université de Genève, Genève, Switzerland

G. A. Payne, North Carolina State University, Raleigh, NC,

U.S.A.

S. C. Peck, John Innes Centre, Norwich, U.K.

V. Penmetsa, University of California, Davis, CA, U.S.A.

X. Perret, Université de Genève, Genève, Switzerland

M. E. Petracek, Oklahoma State University, Stillwater, OK, U.S.A.

C. M. J. Pieterse, Utrecht University, Utrecht, The Netherlands

M. Pirhonen, University of Helsinki, Helsinki, Finland

A. Polle, Forstbotanisches Institut, Gottingen, Busgenweg, Germany

P. Poole, University of Reading, Reading, U.K.

S. Poussier, Kansas State University, Manhattan, KS, U.S.A.

R. Prade, Oklahoma State University, Stillwater, OK, U.S.A.

R. Proctor, USDA ARS MWA NCAUR, Peoria, IL,U.S.A.

D. Prusky, ARO The Volcani Center, Bet Dagan, Israel

A. Pugin, UMR INRA-Université De Bourgogne, Dijon France

A. Puppo, UMR CNRS-UNSA-INRA IPMSV, Sophia-

Antipolis, France

B. Py, CNRS, Marseille, France

J. Rathjen, Colney Lane, Norwich, U.K.

W. Ream, Oregon State University, Corvallis, OR, U.S.A.

B. Reinhold-Hurek, University of Bremen, Bremen, Germany

B. L. Reuhs, Purdue University, West Lafayette, IN, U.S.A.

T. E. Richter, University of California, Davis, CA, U.S.A.

D. Roby, UMR CNRS/INRA, Castanet-Tolosan cedex, France

P. Rodriguez-Palenzuela, Ciudad Universitaria s/n, Madrid, Spain

I. Roldan-Ruiz, Agricultural Research Centrum, Melle, Belgium

M. Romantschuk, University of Helsinki, Lahti, Finland

D. Romero, Universidad Nacional Autonoma de Mexico, Cuernavaca, Morelos, Mexico

K. M. Ramonell, The University of Alabama, Tuscaloosa, AL, U.S.A.

M. Roossinck, Samuel Roberts Noble Foundation Inc, Ardmore, OK, U.S.A.

J. Rose, Cornell University, Ithaca, NY, U.S.A.

J. E. Ruiz-Sainz, Universidad de Sevilla, Sevilla, Spain

G. Saalbach, Risø National Laboratory, Roskilde, Denmark
G. P. C. Salmond, University of Cambridge, Cambridge, U.K.

M. J. D. San Francisco, Texas Technical University, Lubbock, TX, U.S.A.

J. Sanjuan, Estacion Exp Del Zaidin, CSIC, Granada, Spain

M. Schobert, Technische Universitaet Braunschweig, Braunschweig, Germany

J. Schoelz, University of Missouri, Columbia, MO, U.S.A.

P. Schweizer, Institute of Plant Genetics And Crop Plant Research, Gatersleben, Germany

E. Seemueller, Institut fuer Pflanzenschutz im Obstbau, Dosseheim, Germany

J. Shah, Kansas State University, Manhattan, KS, U.S.A.

A. D. Shapiro, Delaware Biotechnology Institute, Newark, DE, U.S.A.

A. Sharon, Tel Aviv University, Tel Aviv, Israel

J. Sherrier, University of Delaware, Newark, DE, U.S.A.

K. Shirasu, John Innes Centre, Norwich, U.K.

T. Sijen, The Netherlands Institute for Developmental Biology, Utrecht, The Netherlands

I. Simko, USDA, ARS, Beltsville, MD, U.S.A.

C. Smart, NYSAES, Cornell University, Geneva, NY, U.S.A.

G. Soberon-Chavez, Universidad Nacional Autonoma de Mexico. Mexico D.F., Mexico

C. Sohlenkamp, Universidad Nacional Autonoma de Mexico, Cuernavaca, Morelos, Mexico

I. E. Somssich, Max-Planck-Institut fuer Zuechtungsforschung, Koeln, Germany

W. Y. Song, University of Florida, Gainesville, FL, U.S.A.

R. V. Sonti, Centre for Cellular and Molecular Biology, Hyderabad, India

H. P. Spaink, Leiden University, Leiden, The Netherlands

P. D. Spanu, Imperial College of Science, Technology and Medicine, London, U.K.

D. Straney, University of Maryland Biotechnology Institute, College Park, MD, U.S.A.

A. Sugio, Kansas State University, Manhattan, KS, U.S.A.

G. Sunter, University of Texas at San Antonio, San Antonio, TX, US.A.

S. Swarup, National University of Singapore, Singapore, Singapore

I. Szankowski, University of Hannover, Sarstedt, Germany

Y. Takano, Kyoto University, Kyoto, Japan

X. Tang, Kansas State University, Manhattan, KS, U.S.A.

C. Thomas, University of East Anglia, Norwich, U.K.

M. Thon, Texas A\&M University, College Station, TX, U.S.A.

J. Ton, Université de Neuchâtel, Neuchâtel, Switzerland U.K.

T. Torto-Alalibo, Virginia Tech, Blacksburg, VA, U.S.A.

F. Trail, Michigan State University, East Lansing, MI, U.S.A.

E. W. Triplett, University of Florida, Gainesville, FL, U.S.A.

S. Tsuyumu, Shizuoka University, Shizuoka, Japan

S. Tucker, University of Arizona, Tucson, AZ, U.S.A.

B. Tudzynski, Westfalische Wilhelms Universite, Muenster, Germany

P. Tudzynski, Westfalische Wilhelms Universite, Muenster, Germany

G. Turgeon, Cornell University, Ithaca, NY, U.S.A.

J. Turner, University of East Anglia, Norwich, U.K.

B. Tyler, Virginia Bioinformatics Institute, Virginia Tech,

Blacksburg, VA, U.S.A.

M. Ullrich, School of Engineering and Science, Bremen, Germany

L. J. Vaillancourt, University of Kentucky, Lexington, KY, U.S.A.

Vol. 18, No. 1, 2005 / 5 
J. P. T. Valkonen, University of Helsinki, Helsinki, Finland A. Van Brussel, Leiden University, Leiden, The Netherlands

G. Van den Ackerveken, University of Utrecht, Utrecht, The Netherlands

R. Van der Hoorn, Wageningen University, Wageningen, The Netherlands

E. van der Vossen, PRI, Wageningen University and Research Centre, The Netherlands

H. van Eck, Wageningen University, Wageningen, The Netherlands

L. C. van Loon, Utrecht University, Utrecht, The Netherlands P. van West, University of Aberdeen, Aberdeen, U.K.

A. van't Slot, Wageningen University, Wageningen, The Netherlands

K. A. VandenBosch, University of Minnesota, St. Paul, MN, U.S.A.

J. Vanderleyden, Katholieke Univ Leuven, Heverlee, Belgium

P. Vinuesa, UNAM-CIFN, Cuernavaca, Morelos, Mexico

G. Walker, MIT, Cambridge, MA, U.S.A.

J. Walton, Michigan State University, East Lansing, MI, U.S.A.
G.-L. Wang, Ohio State University, Columbus OH, U.S.A.

D. Wendehenne, UMR INRA 1088/CNRS 5184/Université de Bourgogne, Dijon, France

M. Whalen, San Francisco State University, San Francisco, CA, U.S.A.

C. Williams, Purdue University, West Lafayette, IN, U.S.A.

V. Williamson, University of California, Davis, CA, U.S.A.

W. Wintermantel, USDA-ARS, Salinas, CA, U.S.A.

D. Wipf, Kirschallee 1, Bonn, Germany

C. Woloshuk, Purdue University, West Lafayette, IN, U.S.A.

J.-R. Xu, Purdue University, West Lafayette, IN, U.S.A.

N. Yalpani, Pioneer Hi-Bred International, Johnston, IA, U.S.A.

B. Yang, Kansas State University, Manhattan, KS, U.S.A.

C.-H. Yang, University of Wisconsin-Milwaukee, Milwaukee, WI, U.S.A.

Y. Yang, University of Arkansas, Fayetteville, AR, U.S.A.

S. Zhang, University of Missouri-Columbia, Columbia, MO, U.S.A.

L.-H. Zwiers, Wageningen University, Wageningen, The Netherlands 